\title{
Réacteurs à hautes densités cellulaires pour la production de solutions concentrées de bactériocines
}

\author{
Patrick Boyaval a*, Parwin Bhugaloo-Vial a, Frédérique Duffes ${ }^{\text {a }}$, \\ Anita Metivier ${ }^{b}$, Xavier Dousset $^{b}$, Didier Marion ${ }^{c}$ \\ ${ }^{a}$ Laboratoire de recherche de technologie laitière, Inra, 65, rue de Saint-Brieuc, \\ 35042 Rennes cedex, France \\ ${ }^{b}$ Laboratoire de microbiologie, Enitiaa, domaine de la Géraudière, 44072 Nantes, France \\ ${ }^{c}$ Laboratoire de biochimie et de technologie des protéines, Inra, BP 1627, \\ 44316 Nantes cedex 03, France
}

\begin{abstract}
Production of concentrated bacteriocin solutions in high cell density bioreactors. Bacteriocins are natural proteinaceous antimicrobial substances produced by microorganisms like lactic acid bacteria to dominate the other microflora. Their use in the food industry as natural preservatives is tightly linked to the emergence of efficient and inexpensive production and purification procedures. The production of an anti-Listeria bacteriocin, named Divercin, by Carnobacterium divergens, has been studied in batch, continuous culture and with viable cells immobilized in alginate gel beads retained in a plug flow bioreactor. The latter system gave the highest volumetric productivity with more than $10^{5} \mathrm{AU} \mathrm{L}^{-1} \mathrm{~h}^{-1}$, which was one hundred times the productivity of the batch system. A new simple (two steps) bacteriocin purification method was developed based on Triton -114 phase partitioning. The pilot production of bacteriocin can then be achieved allowing the exploration of the impact of such additions on the microflora of foodstuffs. (C) Inra/Elsevier, Paris.
\end{abstract}

\section{bacteriocin / immobilized cells / purification / Carnobacterium / Triton X-114}

Résumé - Les bactériocines sont des substances naturelles de nature protéique, produites par des micro-organismes et qui présentent une activité bactéricide ou bactériostatique. L'emploi, dans les industries alimentaires, de bactériocines produites par des bactéries lactiques nécessite des méthodologies de production et de purification extrapolables afin de réaliser des expérimentations pilotes. La production d'une bactériocine active contre Listeria, la Divercine V41, par des cellules entières de Carnobacterium divergens V41, a été étudiée en culture discontinue, en culture continue avec des cellules libres et à l'aide de cellules immobilisées dans des billes d'alginate de calcium placées dans un réacteur piston. Les productivités et les concentrations atteintes en bactériocines ont été comparées. Les cellules immobilisées présentent une bonne productivité avec plus de $10^{5} \mathrm{UA} \mathrm{L}^{-1} \mathrm{~h}^{-1}$, que l'on peut avantageusement comparer à celle du système discontinu :

* Correspondance et tirés à part 
$2,810^{3} \mathrm{UA} \mathrm{L}^{-1} \mathrm{~h}^{-1}$. Une méthode de purification simple, rapide, et bon marché, fondée sur l'emploi d'un détergent, a été développée. La production pilote de bactériocine peut donc être raisonnablement envisagée et des études d'impact, au sein des aliments réalisées. (O) Inra/Elsevier, Paris.

bactériocine / cellules immobilisées / purification / Carnobacterium / triton X114

\section{INTRODUCTION}

La capacité des bactéries lactiques à inhiber la croissance d'autres souches est due à quatre éléments principaux : i) les rejets du catabolisme : les acides organiques qui acidifient le milieu ; ii) la production de métabolites de $1^{\prime} \mathrm{O}_{2}\left(\mathrm{H}_{2} \mathrm{O}_{2}\right)$; iii) les déplétions nutritionnelles engendrées par leur croissance dans le milieu et iv) la production de bactériocines. Ces activités antimicrobiennes peuvent se manifester contre des bactéries comme Salmonella, Listeria, Staphylococcus et Clostridium.

Le rôle des bactéries lactiques en industrie laitière étant considérable, ces bactéries et les bactériocines qu'elles produisent ont fait l'objet de nombreuses études, essentiellement de description moléculaire. La possibilité d'utiliser ces nouvelles substances, naturelles et produites par des souches bactériennes universellement reconnues d'usage alimentaire (GRAS), pour inhiber des flores indésirables comme C. tyrobutyricum et pathogènes comme L. monocytogenes et ainsi diminuer le nombre d'intoxications alimentaires, est constamment mise en avant.

Les quantités de bactériocines obtenues dans ces travaux sont souvent de l'ordre du microgramme. Les productivités sont faibles et surtout les étapes de la purification sont nombreuses et peu ou pas adaptées à l'échelle industrielle. La principale conséquence est que la nisine est la seule bactériocine actuellement produite et uti- lisée industriellement sous une forme non purifiée (Nisaplin, Royaume-Uni).

La mise au point d'une (ou de plusieurs) méthode(s) de production-purification de bactériocines est la principale pierre d'achoppement à une large diffusion de ces molécules dans les industries agricoles et alimentaires (lait, viandes, poissons, etc). Ces petits peptides doivent pouvoir être produits par fermentation, en grandes quantités, forte concentration et à un faible coût. En outre, elles présentent des caractéristiques extrêmement fortes en matière d'hydrophobicité et d'adsorption sur les polymères (et en particulier sur de nombreux plastiques) qui rendent leur concentration et leur purification délicates.

Les bactériocines interagissent avec les membranes des cellules cibles. Elles induisent des pertes de petites molécules telles que des ions, des sucres, de l'ATP ou des acides aminés [4]. Ces peptides hydrophobes présenteraient donc une affinité pour les lipides membranaires. Il paraissait donc intéressant de leur appliquer les méthodes d'extraction les plus performantes employées lors de l'extraction des protéines membranaires. Bordier [3] a montré pour la première fois la pertinence de l'emploi du Triton X 114 pour la purification spécifique et quantitative des protéines transmembranaires.

Dans cette présentation, nous montrerons que la production de bactériocines est envisageable avec des productivités volumiques améliorées et qu'une métho- 
dologie de purification simple peut être appliquée à ces molécules.

\section{MATÉRIEL ET MÉTHODES}

\subsection{Souches, milieux et méthodes de fermentation}

Les souches utilisées dans cette étude sont, pour la souche productrice, Carnobacterium divergens $\mathrm{V} 41$ souche isolée de viscères de poissons [6]. Elle était cultivée à $\mathrm{pH}=6,5$ sur milieu MRS [5] déficient en Tween pour les études de production en batch et en fermentation continue. Un milieu mMRS (MRS sans Tween 80 ni phosphate additionné de $\mathrm{CaCl}_{2}$ $\left(1 \mathrm{~g} \mathrm{~L}^{-1}\right)$ était utilisé pour les expérimentations avec les cellules immobilisées. Carnobacterium piscicola $\mathrm{NCDO} 2762$ et $L$. innocua $\mathrm{F}$. (ENITIAA, Nantes, France) ont été utilisées en tant que souches cibles ou souches sensibles. Ces dernières étaient cultivées sur milieu Elliker (Biokar). Les souches étaient conservées à $-80^{\circ} \mathrm{C}$ dans leur milieu de culture additionné de $15 \%(\mathrm{v} / \mathrm{v})$ de glycérol.

Les cultures batch et continue étaient réalisées à $30^{\circ} \mathrm{C}$ sous agitation $(80 \mathrm{rpm})$ dans des bioréacteurs de $2 \mathrm{~L}$ (Set 2M, SGI, Toulouse, France). Le $\mathrm{pH}$ était régulé à 6,5 par addition automatique de $\mathrm{NaOH} 5 \mathrm{M}$. La préparation des cellules immobilisées et leur mise en cuvre suivaient la procédure décrite dans Boyaval et Goulet [2].

La biomasse était déterminée par mesure de densité optique corrélée à des mesures de masse sèche et détermination du nombre de cellules viables après étalement sur milieu MRS et incubation 48 heures, à $30^{\circ} \mathrm{C}$, en anaérobiose (anaerocult, Merck, Germany). L'activité bactériocinique était quantifiée selon la méthode de la dilution critique [1] et exprimée en unités arbitraires par millilitre de solution (UA mL-1).

\subsection{Purification de la bactériocine}

Le Triton X114 (Fluka, Germany) était ajouté à $4{ }^{\circ} \mathrm{C}$ à un surnageant de culture, pour atteindre une concentration de $2 \%$. Le pH était ajusté à 5,5 , avec $\mathrm{HCl} 1 \mathrm{~mol} \mathrm{~L}^{-1}$ et l'ensemble était porté à $25-30^{\circ} \mathrm{C}$. La séparation de phase était totale après 1 à 2 heures. La phase inférieure, riche en détergent, était collectée, lavée avec de l'eau désionisée froide contenant du Triton $(0,2 \%, \mathrm{~m} / \mathrm{v})$, et l'opération était répétée deux fois. La phase riche en Triton finalement obtenue était diluée 10 fois avec de l'eau désionisée et déposée sur une résine cationique de $50 \mathrm{~mL}$ (Cellulose Ion Exchanger, CM-C500, Amicon, France). La colonne était ensuite lavée avec $50 \mathrm{~mL}$ d'eau désionisée froide. L'élution de la bactériocine était réalisée par un gradient : tampon A (eau désionisée) et tampon B $\left(\mathrm{NaCl} 0,7 \mathrm{~mol} \mathrm{~L}^{-1}\right.$ ) (FPLC Pharmacia, France) : 0 à 150 minutes : 0 à $70 \%$ tampon $\mathrm{B} ; 150$ à 152 minutes : 70 à $100 \%$ tampon $\mathrm{B} ; 152$ à 180 minutes : $100 \%$ tampon $B$ et 180 à 280 minutes : $100 \%$ tampon A. La pureté des échantillons était examinée par HPLC en phase inverse, comme précédemment décrit [1].

\section{RÉSULTATS ET DISCUSSION}

\subsection{Fermentations}

\subsubsection{Fermentations Batch}

La biomasse atteint les $2,1 \pm 0,05 \mathrm{~g} \mathrm{~L}^{-1}$ après 45 heures et la concentration en divercine $10^{5} \mathrm{UA} \mathrm{mL}^{-1}$. La productivité volumique est de $2200 \mathrm{UA} \mathrm{mL}^{-1} \mathrm{~h}^{-1}$.

\subsubsection{Fermentation continue}

La biomasse la plus élevée, 4,2 4 $0,1 \mathrm{~g} \mathrm{~L}^{-1}$, était obtenue pour un taux de dilution de $0,03 \mathrm{~h}^{-1}$. Une augmentation de la production de bactériocine était observée jusqu'à des taux de dilution de $0,2 \mathrm{~h}^{-1}$ avec un maximum de $2,0510^{5} \mathrm{UA} \mathrm{mL}^{-1}$. Une productivité volumique maximale de $4,110^{4} \mathrm{UA} \mathrm{mL}^{-1} \mathrm{~h}^{-1}$ était observée pour une biomasse de $2,2 \pm 0,05 \mathrm{~g} \mathrm{~L}^{-1}$.

\subsubsection{Fermentation continue avec cellules immobilisées}

Une augmentation régulière de la productivité volumique était observée jusqu'à un taux de dilution de $2,0 \mathrm{~h}^{-1}$, où elle attei- 
Tableau I. purification avec le TX114 d'un surnageant de culture.

Table I. TX114 purification of a culture supernatant.

\begin{tabular}{lccccc}
\hline $\begin{array}{l}\text { Étapes de } \\
\text { purification }\end{array}$ & $\begin{array}{c}\text { Volume } \\
(\mathrm{mL})\end{array}$ & $\begin{array}{c}\text { Protéines } \\
(\mathrm{mg})\end{array}$ & $\begin{array}{c}\text { Activité } \\
\text { totale } \\
(\mathrm{UA})\end{array}$ & $\begin{array}{c}\text { Activité } \\
\text { spécifique } \\
(\mathrm{UA} / \mathrm{mg})\end{array}$ & $\begin{array}{c}\text { Rendement } \\
\text { protéique } \\
(\%)\end{array}$ \\
\hline $\begin{array}{l}\text { Surnageant } \\
\text { de culture }\end{array}$ & 3950 & $3,210^{4}$ & $1,6210^{9}$ & $510^{4}$ & 100 \\
$\begin{array}{l}\text { Triton X-114 } \\
\text { partition } \\
\text { de phase }\end{array}$ & 280 & 40,88 & $\mathrm{ND}$ & $\mathrm{ND}$ & 0,13 \\
$\begin{array}{l}\text { Chromatographie } \\
\text { échange de } \\
\text { cations }\end{array}$ & 90 & 14,22 & $910^{9}$ & $6,410^{8}$ & 0,04 \\
\hline
\end{tabular}

gnait $10^{5} \mathrm{UA} \mathrm{mL} \mathrm{m}^{-1} \mathrm{~h}^{-1}$. Une légère fuite cellulaire était observée à partir de $0,8 \mathrm{~h}^{-1}$.

L'utilisation d'une procédure de fermentation mettant en oeuvre des cellules immobilisées dans des billes d'alginate permet aisément de multiplier par 100 la productivité volumique de la production de divercine par Carnobacterium divergens V41. Dès lors, des méthodes de purification pilote peuvent être envisagées.

\subsection{Purification de la bactériocine}

La nouvelle procédure de purification associe une partition de phase et une chromatographie d'échange de cations. La totalité de la bactériocine, en solution dans le milieu de culture, se trouve concentrée par cette procédure. La divercine, en solution de chlorure de sodium $(0,2 \mathrm{M})$, est directement utilisable pour des études structure/fonction ou pour son emploi en industrie alimentaire. Aucune trace de détergent n'est détectable après cette deuxième étape. L'activité spécifique est augmentée en moyenne d'un facteur 12000 , tandis que l'activité totale augmente d'un facteur 5, probablement par les phénomènes d'agrégation-désagrégation de cette molécule hydrophobe (tableau I). La pureté de la bactériocine est supérieure à $95 \%$. La solution se conserve plusieurs mois à $4^{\circ} \mathrm{C}$ et au moins une année à $-20^{\circ} \mathrm{C}$. Il est à noter que ces molécules s'adsorbent rapidement sur les plastiques et que les étapes de purification et de conservation doivent s'effectuer à l'aide de contenants en verre.

L'adéquation de cette méthode à d'autres bactériocines à été validée sur la nisine et la mésentéricine Y105.

\section{RÉFÉRENCES}

[1] Bhugaloo-Vial P., Dousset X., Metivier A., Sorokine O., Anglade P., Boyaval P., Marion D., Purification and amino acid sequences of piscicocins V1a and V1b, two class IIa bacteriocins secreted by Carnobacterium piscicola V1 that display significantly different levels of specific inhibitory activity, Appl. Environ. Microbiol. 62 (1996) 4410-4416.

[2] Boyaval P., Goulet J., Optimal conditions for production of lactic acid from cheese whey permeate by $\mathrm{Ca}$-alginate-entrapped Lactobacillus helveticus, Enzyme Microbiol. Technol. 10 (1988) 725-728.

[3] Bordier C., Phase separation of integral membrane proteins in Triton X114 solution, J. Biol. Chem. 25 (1981) 1604-1607. 
[4] Chen Y., Montville T.J., Efflux of ions and ATP depletion induced by pediocin PA-1 are concomitant with cell death in Listeria monocytogenes Scott A, J. Appl. Bacteriol. 79 (1995) 684-690.

[5] De Man J.C., Rogosa M., Sharpe E., A medium for the cultivation of Lactobacilli, J. Appl. Bacteriol. 23 (1960) 130-135.
[6] Pilet M.F., Dousset X., Barre R., Novel G., Desmazeaud M.J., Piard J.C., Evidence of two bacteriocins produced by Carnobacterium piscicola V1 and Carnobacteriocin divergens $\mathrm{V} 41$ isolated from fish and active against Listeria monocytogenes, J. Food Prot. 58 (1995) 256-262. 